\title{
Recent progress in quantum and nonlinear optical lithography
}

\author{
ROBERT W. BOYD*† and SEAN J. BENTLEY† \\ $\dagger$ Institute of Optics and Department of Physics and Astronomy, \\ University of Rochester, Rochester, New York 14627, USA \\ Department of Physics, Adelphi University, \\ Garden City, New York 11530, USA
}

(Received 4 March 2005)

\begin{abstract}
We review the status of the field of quantum lithography, that is, the use of quantum-mechanical effects to write lithographic features with resolution finer than that achievable according to the Rayleigh criterion. In particular, we first review the original quantum lithography proposal by Boto et al., and we then describe the status of research aimed at realizing this process.
\end{abstract}

Several proposals have been put forward in recent years for methods to increase the spatial resolution of lithographic systems. These methods are of enormous potential practical importance because, for instance, they could be used to increase the density of electronic gates on a silicon chip. Several years ago Boto et al. [1] proposed to use quantum effects to increase the resolution of the lithographic process. They showed theoretically that a resolution $N$ times greater than the Rayleigh resolution limit [2] could be achieved by making use of $N$ entangled photons in the incident quantum state. This idea is illustrated in figure 1 for the case $N=2$. Here a laser beam falls on to a nonlinear mixing crystal in which parametric downconversion occurs, producing two entangled photons. This photon pair then falls on to a 50-50 beam splitter. As is well known [3], the output of the interferometer under these circumstances is the superposition state $|2,0\rangle+|0,2\rangle$, where the notation is such that $|n, m\rangle$ denotes a state in which $n$ photons are in the upper output port of the interferometer and $m$ photons are in the lower port. Note that there is no way in which one photon can leave in each of the output ports. These two output beams are then made to interfere on a recording medium that responds by means of two-photon absorption. Fringes are formed in the medium by means of the interference between the probability amplitudes for two-photon absorption with the photon pair taking either the upper or the lower pathway. Each of these probability amplitudes depends on path length $L$ as $\exp (2 \mathrm{i} k L)$, where the factor of 2 occurs because each of the two photons acquires the phase shift $k L$. The fringe spacing is thus twice as fine as that given by the normal interference patterns between the two waves. In many ways, the enhanced

*Corresponding author. Email: boyd@optics.rochester.edu 


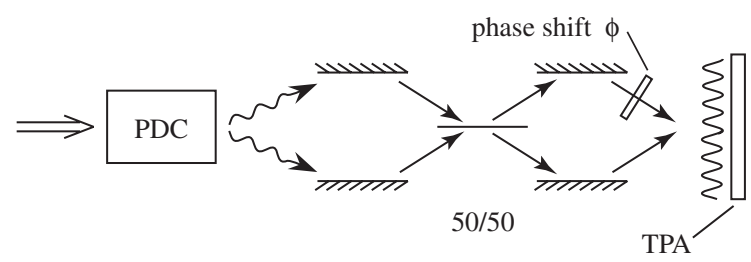

Figure 1. Schematic experimental set-up for quantum lithography and quantum microscopy: PDC, parametric downconverter. This set-up can be used to record interference fringes with a spacing twice as dense as predicted by classical interference.

resolution can be understood from the point of view that the de Broglie wavelength of a quantum state consisting of two entangled photons is half the classical wavelength associated with either photon $[4,5]$.

We have drawn figure 1 with the beams striking the recording medium at oblique incidence, although in practice the beams would be directed towards the medium at near-grazing incidence to achieve the finest fringe spacing possible. Note that the ability to write small structures scales with the order $N$ of the interaction. That is, if $N$ photons are entangled and the recording medium responds by $N$-photon absorption, features of size $\lambda / 2 N$ can be written into the material. Although the initial idea stressed the writing of small features, we note the intimate relation between writing small features and observing small features. This basic interaction could thus be used in reverse to build microscopes that can resolve features much smaller than $\lambda / 2$. This ability could be of immense importance in fields such as biomedicine, where to prevent damaging the tissue under investigation it is not possible to use short wavelengths to obtain increased spatial resolution.

Two problems stand in the way of the successful implementation of quantum lithography:

(i) the development of intense sources of entangled photons that can be used as the excitation;

(ii) the development of recording media that respond by $N$-photon absorption.

We note that these two problems are intimately intertwined. For instance, if the excitation source becomes so intense that each field mode contains more than one photon, the nature of the quantum interference leading to increased resolution can become degraded, leading to a partial (but not complete!) loss of contrast and/or resolution [6-8]. Moreover, the very nature of an entangled light field can lead directly to an increase in the efficiency of $N$-photon absorption, as the photons tend to arrive at the recording medium $N$ at a time. Some work has been carried out to analyse this situation theoretically $[9,10]$ and the effect has been observed in the laboratory [11], but more theoretical and experimental work on this point remains to be done. From a laboratory point of view, it seems at present that the best candidate materials for $N$-photon lithography include standard ultraviolet lithographic materials excited in the visible or a 
broad-bandgap material such as poly(methyl metacrylate) excited by multiphoton absorption.

At least two proof-of-principle experiments have been reported which display various aspects of the quantum lithography proposal. D'Angelo et al. [12] have made use of a parametric downconverter to create entangled photons and mimicked the process of two-photon absorption using coincidence circuitry. Bentley and Boyd [13] adopted a different approach, using classical light with specially tailored coherence properties to mimic the unusual quantum statistics of entangled photons. This work followed an earlier suggestion of Ooki et al. [14]. We shall next describe this procedure more fully.

To understand this procedure for obtaining enhanced resolution, we consider the exposure induced into a lithographic material when two light fields interfere on the surface of the material, as illustrated in figure 2. If the recording material responds by means of normal one-photon absorption, the exposure rate of the material will be proportional to

$$
E=1+\cos (q x)
$$

where $q=2(\omega / c) \sin \theta$ is the wave vector magnitude of the interference pattern of the light waves. In the proposal by Boto et al., in which two entangled photons are used and the recording medium acts by means of two-photon absorption, the exposure rate is given by

$$
E=1+\cos (2 q x)
$$

as noted above, the fringe spacing here is twice as fine as in equation (1). Finally note that, if an intensity pattern of the form of equation (1) falls on to a medium that operates by means of two-photon absorption, the exposure rate will be proportional to

$$
\begin{aligned}
E & =[1+\cos (q x)]^{2} \\
& =1+2 \cos (q x)+\cos ^{2} q x \\
& =\frac{3}{2}+2 \cos (q x)+\frac{1}{2} \cos (2 q x)
\end{aligned}
$$

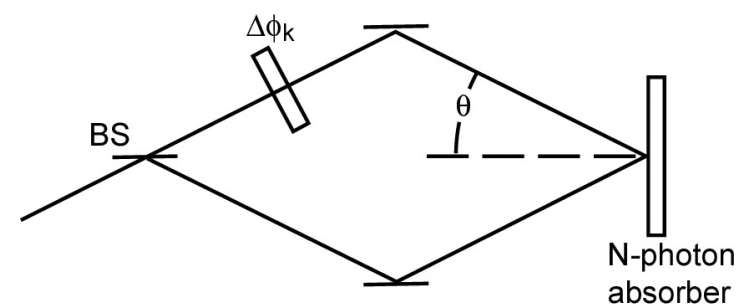

Figure 2. Schematic illustration of the new method for achieving enhanced resolution using classical light: BS, beam splitter. In this technique, $M$ individual interference patterns are summed together, with a phase shift of $2 \pi / M$ introduced between successive interference patterns. In this manner, unwanted spatial Fourier components in the interference pattern are forced to average to a constant value. 
Note that the final form of equation (3) differs from the desired form (2) in two ways. Firstly, the spatially uniform term $\frac{3}{2}$ is three times larger than desired. Thus, the contrast of the fringe pattern is reduced. Secondly, there is an additional unwanted term, with spatial frequency $q$ (rather than $2 q$ ). However, as we see from figure 2, we can make this term average to a constant by adding the results of two exposures with a phase difference of $\pi \mathrm{rad}$ introduced into one of the beams between the exposures. It is further shown in [13] that this method scales to an arbitrarily large resolution enhancement $M$ simply by averaging $M$ patterns with an applied relative phase of $2 \pi / M$, and using an order of nonlinearity $N$ at least as large as $M$. Moreover, for $N$ considerably greater than $M$ the resulting fringe pattern possesses high visibility.

The experimental procedure used to study this effect is shown in figure 3 . We noted above the difficulty in obtaining good lithographic materials that operate by means of two-photon absorption. For this reason, in this study we simulated two-photon detection by making use of the process of second-harmonic generation

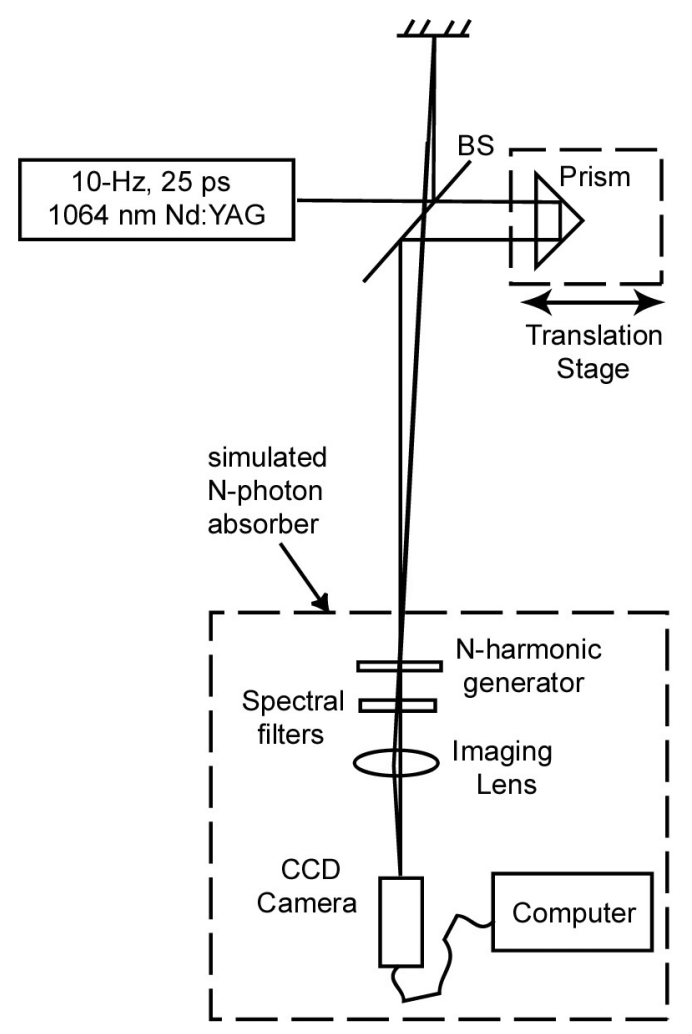

Figure 3. Set-up of the experiment used to demonstrate enhanced resolution by phase averaging with classical light. $N$-photon absorption is mimicked using $N$ th-harmonic generation and a charge-coupled device (CCD) camera: BS, beam splitter; Nd: YAG, neodymium-doped yttrium aluminium garnet. 

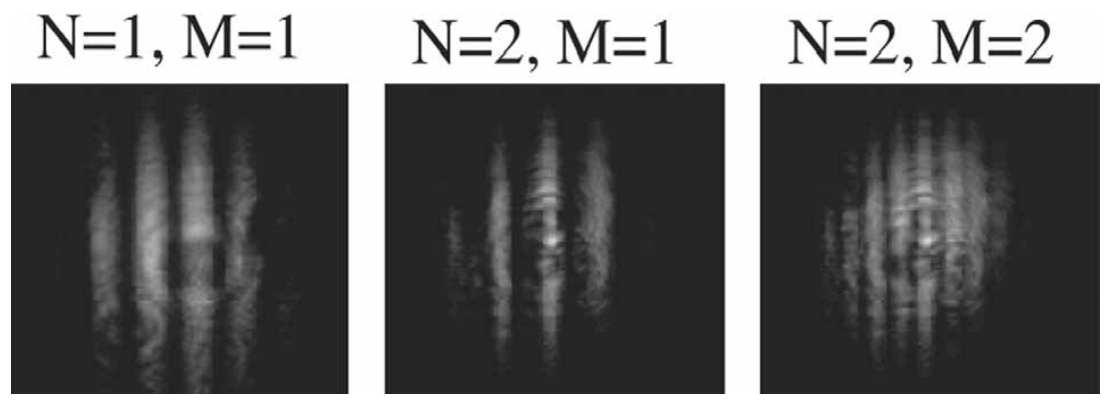

Figure 4. Measured intensity distributions for $M=N=1$, for $M=1, N=2$, and for $M=N=2$. Note that the first two patterns have the same period (because $M=1$ ) but that the fringes are sharper for larger $N$. Note also the doubling of the fundamental frequency in the rightmost image.

followed by linear detection on a charge-coupled device camera. Some of the results of this study are shown in figure 4 . We see (reading from left to right) normal interference fringes, sharpened fringes by using simulated two-photon detection but not using phase averaging and finally a doubling of the fringe frequency by using phase averaging and simulated two-photon detection.

In summary, we feel that quantum lithography (as initially proposed by Boto et al. [1]) has a very good chance of becoming a reality, especially as new sensitive multiphoton lithographic materials are being developed. We have also seen that classically simulated quantum lithography and other forms of nonlinear optical lithography $[15,16]$ may be realistic alternative approaches, and ones that are much more readily implemented. Crucial to the practical implementation of these methods will be the development of methods for writing arbitrary patterns rather than just periodic fringe patterns. Some success along these lines has been reported already [17-19]. The fact that quantum lithography can be mimicked classically is reminiscent of recent work aimed at determining which features of ghost imaging can be realized with classical sources [20-25]. One conclusion to be drawn is that there is still much work to be done in the field of quantum imaging to delineate the quantum-classical frontier.

\section{Acknowledgments}

We acknowledge valuable discussions with R. Bennink, H. J. Chang, K. Dolgaleva, J. Dowling, S. Hocde, Anand Jha, M. O'Sullivan-Hale, H. Shin and P. Zerom. This work is supported by the US Army Research Office.

\section{References}

[1] N. Boto, P. Kok, D.S. Abrams, et al., Phys. Rev. Lett. 852733 (2000).

[2] Lord Rayleigh, Phil. Mag. 8261 (1879). 
[3] C.K. Hong, Z.Y. Ou and L. Mandel, Phys. Rev. Lett. 592044 (1987).

[4] E.J.S. Fonseca, C.H. Monken and S. Pádua, Phys. Rev. Lett. 822868 (1999).

[5] K. Edamatsu, R. Shimizu and T. Itoh, Phys. Rev. Lett. 89213601 (2002).

[6] G.S. Agarwal, R.W. Boyd, E.M. Nagasako, et al., Phys. Rev. Lett. 861389 (2001).

[7] E.M. Nagasako, S.J. Bentley, R.W. Boyd, et al., Phys. Rev. A 64043802 (2001).

[8] E.M. Nagasako, S.J. Bentley, R.W. Boyd, et al., J. Mod. Optics 49529 (2002).

[9] J. Javanainen and P.L. Gould, Phys. Rev. A 415088 (1990).

[10] J. Perina, B.E.A. Saleh and M.C. Teich, Phys. Rev. A 573972 (1998).

[11] N.P. Georgiades, E.S. Polzik, K. Edamatsu, et al., Rev. Rev. Lett. 753426 (1995).

[12] M. D’Angelo, M.V. Chekhova and Y. Shih, Phys. Rev. Lett. 87013602 (2001).

[13] S.J. Bentley and R.W. Boyd, Optics Express 125735 (2004).

[14] H. Ooki, M. Komatsu and M. Shibuya, Jap. J. Appl. Phys. 33 L177 (1994).

[15] E. Yablonovitch and R.B. Vrijen, Opt. Engng 38334 (1999).

[16] D. Korobkin and E. Yablonovitch, Opt. Engng 411729 (2002).

[17] S.R.J. Brueck, S.H. Zaidi, X. Chen, et al., Microelectron. Engng 42145 (1998).

[18] P. Kok, A.N. Boto, D.S. Abrams, et al., Phys. Rev. A 63063407 (2001).

[19] G. Bjork, L.L. Sanchez-Soto and J. Soderholm, Phys. Rev. Lett. 864516 (2001).

[20] T.B. Pittman, Y.H. Shih, D.V. Strekalov, et al., Phys. Rev. A 52 R32 429 (1995)

[21] A.F. Abouraddy, B.E.A. Saleh, A.V. Sergienko, et al., Phys. Rev. Lett. 87123602 (2001).

[22] R.S. Bennink, S.J. Bentley and R.W. Boyd, Phys. Rev. Lett. 89113601 (2002).

[23] A. Gatti, B. Brambilla and L.A. Lugiato, Phys. Rev. Lett. 90133603 (2003).

[24] R.S. Bennink, S.J. Bentley, R.W. Boyd, et al., Phys. Rev. Lett. 92033601 (2004).

[25] A. Valencia, G. Scarcelli, M. D’Angelo, et al., Phys. Rev. Lett. 94063601 (2005). 